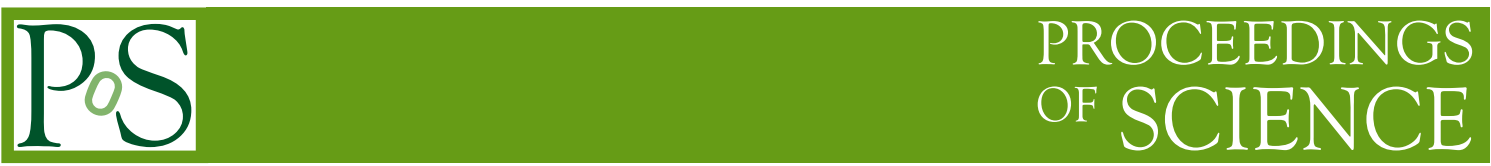

\title{
Screening at finite temperature and density
}

\author{
Olaf Kaczmarek* \\ Fakultät für Physik, Universität Bielefeld, D-33615 Bielefeld, Germany \\ E-mail: okacz@physik.uni-bielefeld.de
}

We present lattice QCD results on heavy quark free energies, extract from its temperature dependence entropy and internal energy contributions, and discuss the onset of medium effects that lead to screening of static quark-antiquark sources in a thermal medium. Most results are obtained in $(2+1)$-flavour QCD on a line of constant physics with almost realistic quark masses and compared to previous results from 2-flavor QCD as well as pure gauge theory. Furthermore, we discuss results on the density dependence of screening masses that have been obtained using a leading order Taylor expansion in the baryon chemical potential.

Critical Point and Onset of Deconfinement 4th International Workshop

July 9-132007

GSI Darmstadt,Germany

\footnotetext{
* Speaker.
} 


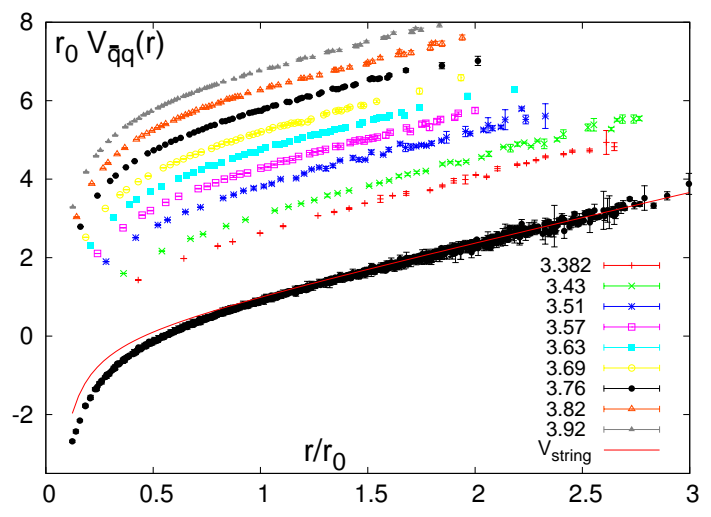

Figure 1: Un-renormalized zero temperature potentials for different values of $\beta$ and renormalized overall $T=0$-potential (lower black points) in units of $r_{0}$. The solid line shows the string potential as discussed in the text.

\section{Introduction}

In-medium properties of heavy quarks and heavy quark bound states are of fundamental interest for the understanding of strongly interacting matter at high temperatures and densities probed in current and future heavy ion collision experiments at RHIC, FAIR and LHC. The temperature dependence of heavy quark free energies, potential energies as well as screening masses and radii are important ingredients for studies on strongly coupled quark gluon plasma, possible existence of heavy quark bound states in the QGP as well as on transport properties of heavy quarks in the high temperature phase.

We present preliminary results on heavy quark free energies [1] that are based on an analysis of gauge field configurations generated by the RBC-Bielefeld collaboration in (2+1)-flavor QCD for the calculation of the QCD equation of state [2]. The pion mass is about $220 \mathrm{MeV}$ and the strange quark mass is adjusted to its physical value. The calculations were performed with improved staggered fermions on lattices with temporal extent $N_{\tau}=4$ and 6 accompanied by high statistics zero temperature calculations to set the scale and to extract the zero temperature potential and corresponding renormalization constants.

We perform a renormalization of the finite temperature heavy quark free energies and of the Polyakov loop using the renormalization constants obtained at zero temperature. We will discuss the relation between heavy quark free energies, entropy and internal energy contributions and analyze their critical behavior in the transition region. The temperature dependence of the interaction between static quark anti-quark pairs will be analyzed in terms of, in general distance and temperature dependent, running couplings and screening masses. A preparatory study of the density dependence of screening masses in 2-flavor QCD with large quark masses indicates that non-perturbative effects in the behavior of screening is dominated by the gluonic sector.

\section{Zero temperature potential}

In fig. 11 we show the zero temperature potential, $V_{\bar{q} q}(r)$, in units of the distance scale $r_{0}$ for various values of the coupling $\beta$ corresponding to different values of the lattice cut-off varying 

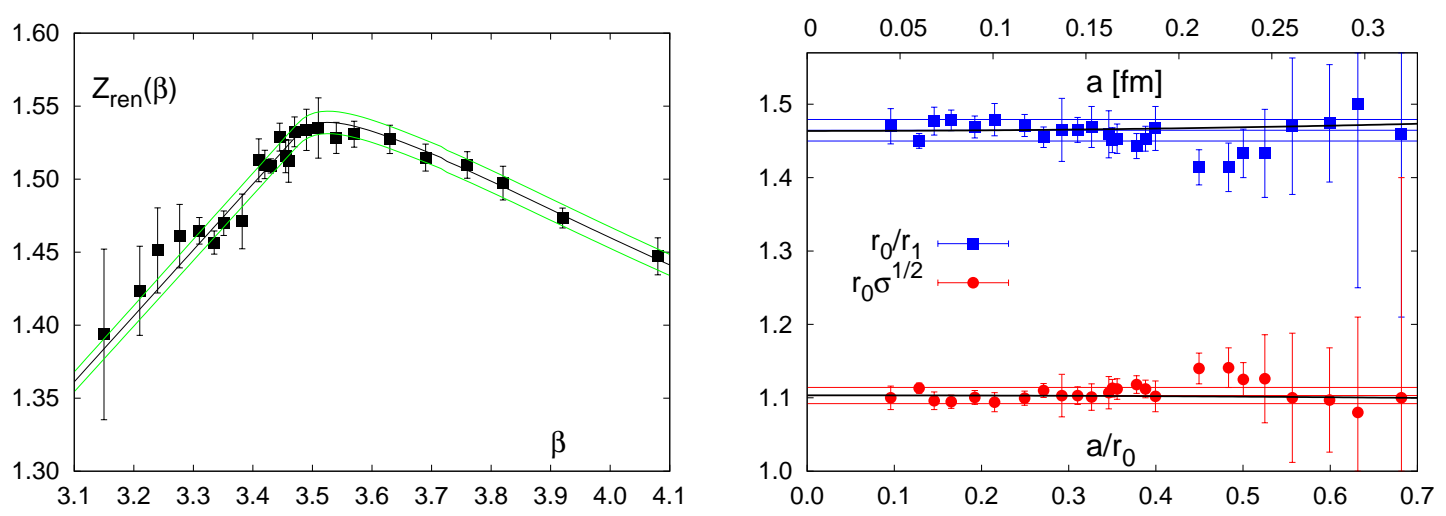

Figure 2: Renormalization constants, $Z_{\mathrm{ren}}(\beta)$, obtained from the matching of the zero temperature potentials (left). Dimensionless combinations, $r_{0} / r_{1}$ and $r_{0} \sqrt{\sigma}$. The lower axis denotes the scale $\left(r_{0} / a\right)^{-1}$ and the upper axis the lattice cut-off in physical units. The lines are explained in the text (right).

from $a \simeq 0.3 \mathrm{fm}$ down to $a \simeq 0.05 \mathrm{fm}$. The scale $r_{0} / a$ is define by the slope of the potential,

$$
\left(r^{2} \frac{d V_{\bar{q} q}(r)}{d r}\right)_{r=r_{0}}=1.65
$$

and can be used to convert to physical scales with a value $r_{0}=0.469(7) \mathrm{fm}$ [3]. Additional parameters obtained from the slope of $V_{\bar{q} q}(r)$ are the string tension, $\sigma a^{2}$, and $r_{1} / a$ defined by replacing 1.65 by 1.0 in 2.1). The dimensionless combinations of these parameters displayed in fig. 2 show only small cut-off effect, e.g. $r_{0} \sqrt{\sigma}$ stays constant in the entire range of couplings in which the lattice spacing changes by a factor of 6 . Using a quadratic fit Ansatz for $a \leq 0.15 \mathrm{fm}$ we obtain $r_{0} \sqrt{\sigma}=1.1034(40)$ and $r_{0} / r_{1}=1.4636(60)$. For both ratios we observe that $\mathscr{O}\left(a^{2}\right)$ corrections are small.

The potentials as calculated on the lattice are ultraviolet divergent and need to be renormalized. We have matched all potentials to a common value at large distances, $r / r_{0}=1.5$, taken to be identical to the large distance string potential which in units of $r_{0}$ is given by

$$
r_{0} V_{\text {string }}\left(r / r_{0}\right)=-\frac{\pi}{12 r / r_{0}}+\left(\sigma r_{0}^{2}\right) \frac{r}{r_{0}},
$$

where we have used the value $r_{0} \sqrt{\sigma}$ quoted above. The result of this renormalization is shown in the lower part of fig. 1. The good matching of all potential data over the whole distance range again shows that cut-off effects are small in this observable.

The matching procedure provides renormalization constants for the static quark potential which we will use for the renormalization of Polyakov loops and heavy quark free energies at finite temperatures. They are defined as $Z_{\mathrm{ren}}(\beta)=\exp ((c(\beta) a) / 2)$, where $c(\beta)$ denotes the constant shift for the corresponding zero temperature potential. Already note here that no additional divergencies arise when going from zero to finite temperatures. The solid line in fig. 11 shows the string potential. Note that the deviations of the potential $V_{\bar{q} q}(r)$ from $V_{\text {string }}(r)$ at small distances shows the effect of the QCD running coupling while the Coulombic term in the string potential stems from large distance corrections in the string model. Furthermore we do not observe string breaking here which is due the operator used in our analysis and the limited distance range analyzed here. 

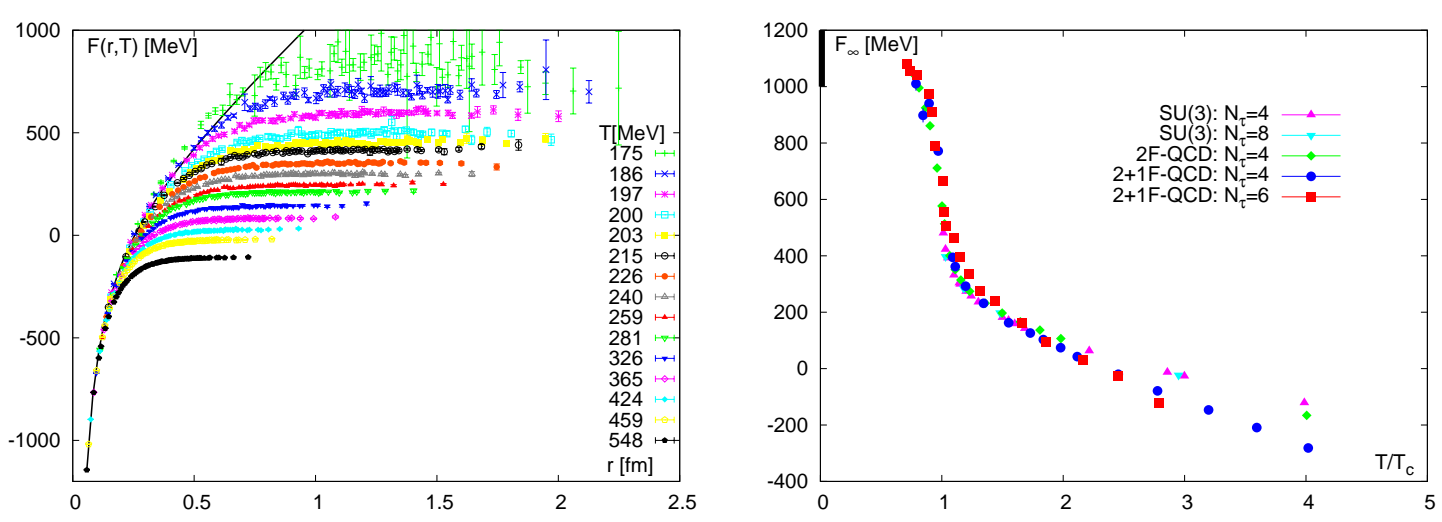

Figure 3: Renormalized heavy quark free energies, $F_{1}(r, T)$, for different values of the temperature (left). The solid line shows the zero temperature potential $V_{1}(r)$. Asymptotic values for the heavy quark free energies (right), $F_{1}(r=\infty, T)$, for $(2+1)$ flavors compared to earlier results for pure gauge theory and 2flavor [4, 5, 6].

\section{Heavy quark free energies}

The heavy quark free energy of a static quark-antiquark pair in a color singlet state separated by distance $r$ is defined by

$$
\frac{F_{1}(\vec{r}, T)}{T}=-\log \left(\operatorname{Tr}\left(L_{\mathrm{ren}}(\overrightarrow{0}) L_{\mathrm{ren}}(\vec{r})\right)\right),
$$

where $L_{\text {ren }}$ is the renormalized Polyakov loop,

$$
L_{\mathrm{ren}}(\vec{x})=\left(Z_{\mathrm{ren}}(\beta)\right)^{N_{\tau}} L_{\mathrm{bare}}=\prod_{x_{0}=0}^{N_{\tau}-1} Z_{\mathrm{ren}}(\beta) U_{\left(\vec{x}, x_{0}\right), 0} .
$$

The operator used in (3.1) in general is gauge dependent and we fix to Coulomb gauge according to arguments based on [7, 8]. As already noted we are using the renormalization constants, $Z_{\text {ren }}(\beta)$, obtained at zero temperature for the renormalization of the Polyakov loop. In this way also the heavy quark free energies are properly renormalized, which is evident from fig. 3 (left) where $F_{1}(r, T)$ for different temperatures is shown. The solid line is the zero temperature potential $V_{\bar{q} q}(r)$.

The free energies become temperature independent at small separations and coincide with $V_{\bar{q} q}(r)$ showing the correct renormalization. At larger distances temperature effects set in, below $T_{c}$ due to string breaking and above $T_{c}$ due to screening of the static sources in the thermal deconfined medium. The onset of this temperature effects is shifted towards smaller distances with increasing temperature. The asymptotic values, $F_{1}(r=\infty, T)$, are show in fig. 3 (right) compared to previous results from quenched and 2-flavor QCD with larger quark masses [4, 5, 6]. The qualitative behavior is comparable in all those theories. Note that $F_{\infty}$ is infinite for the quenched theory below the critical temperature. With dynamical quarks below the critical temperature $F_{\infty}$ is already close to the value estimated from zero temperature (indicated by the black band), it shows a strong decrease around $T_{c}$ and a quite linear behavior at high temperatures. This temperature dependence already indicates that entropy contributions play an important role especially around the transition temperature. 

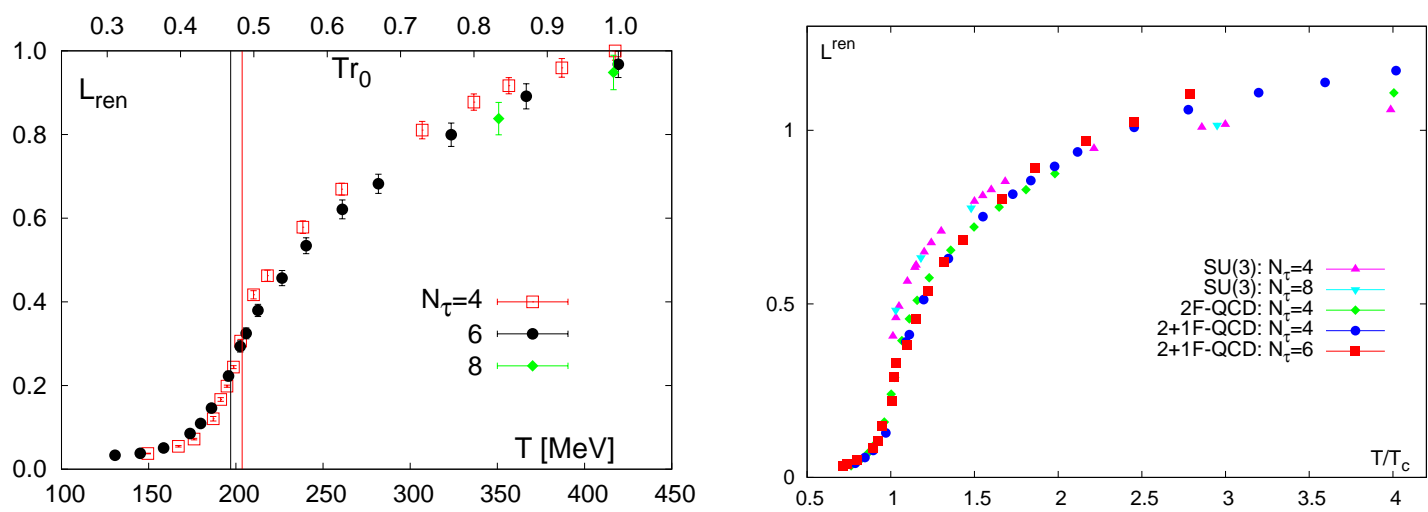

Figure 4: The renormalized Polyakov loop for different temporal lattice extents, $N_{\tau}=4,6$ and 8 (left). The vertical lines show the location of the transition temperature determined in [2] on lattices with temporal extent $N_{\tau}=4$ (right line) and $N_{\tau}=6$ (left line), respectively. Comparison to earlier results (right) for pure gauge theory and 2-flavor [4, 5, 6].

\section{Renormalized Polyakov loop}

As already discussed in the previous section, the renormalization constants obtained at zero temperature can be used for the renormalization of the Polyakov loop at finite temperature according to (3.2). Due to the cluster property this renormalization is equivalent to a definition of $L_{\text {ren }}$ using the asymptotic value of the heavy quark free energy, $F_{\infty}(T)=F_{1}(r=\infty, T)$, i.e. $L_{\text {ren }}=\exp (-F(r=\infty, T) / 2 T)$. While $L_{\text {ren }}$ is zero below the critical temperature in pure SU(3) gauge theory and has a finite gap at $T_{c}$ due to the first order transition in this theory, it is non-zero in QCD with dynamical quarks even in the low temperature phase. Although the temperature dependence is continuous here, $L_{\mathrm{ren}}$ shows a pronounced rise around the transition region from small to large values in the high temperature phase.

We note that the most rapid change is in good agreement with the region where the chiral condensate as well as bulk thermodynamic quantities, e.g. energy and entropy densities change most rapidly [2]. Furthermore the cut-off dependence of $L_{\text {ren }}$ on lattices with temporal extent $N_{\tau}=4$ and 6 is small, which is in agreement with results obtained in studies of $L_{\mathrm{ren}}$ in pure SU(3) gauge theories [5, 9]. The large cut-off dependence observed in a study with the 1-link stout smeared staggered action used in [10] mainly seems to arise from the cut-off dependence of the zero temperature observable $\left(f_{K}\right)$ used to set the temperature scale. The fact that the renormalized Polyakov loop becomes larger than one at high temperatures was already observed in the quenched theory and is predicted by perturbation theory where the high temperature limit, $L_{\mathrm{ren}}(T=\infty)=1$, is reached from above [11, 12]. Note that the renormalized Polyakov loop as defined in (3.2) is no longer a SU(3) matrix.

\section{Entropy and internal energy contributions}

The temperature dependence of the heavy quark-antiquark free energy at asymptotic large distances, $F_{\infty}(T)$, already indicated that entropy contributions play an important role especially in the vicinity of the transition region. Thus it becomes important for discussions on strongly coupled 

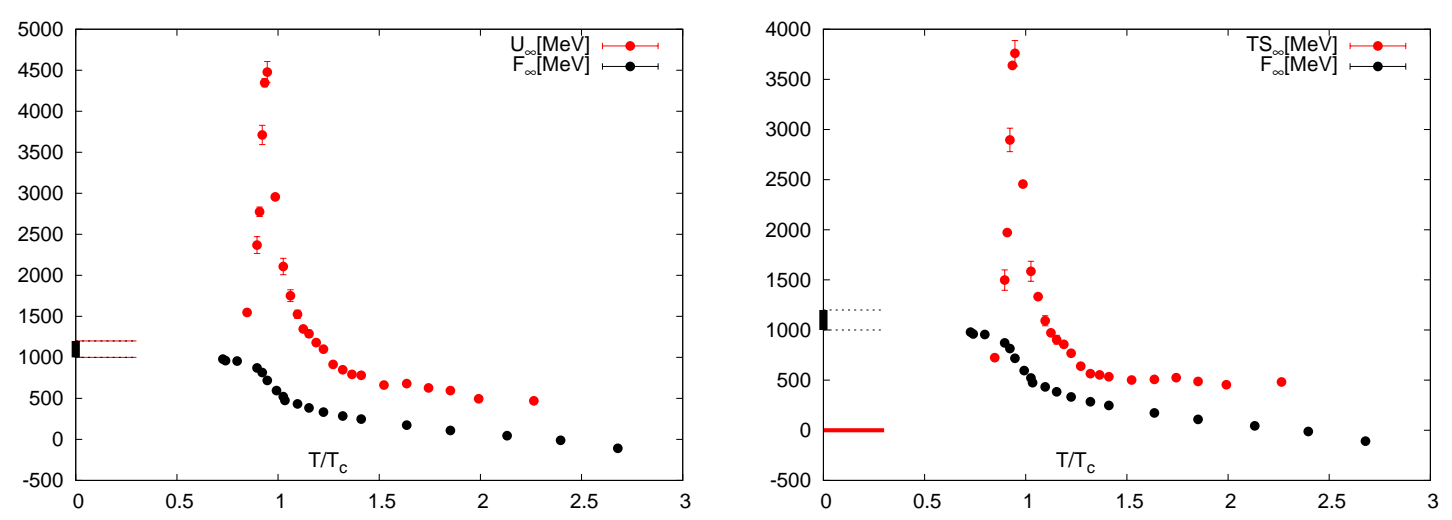

Figure 5: Asymptotic large distance values for internal energy (left) and entropy (right) compared to heavy quark free energies. The left band indicates the zero temperature limit of the energy and the red line shows the vanishing $T=0$ limit for $T S_{\infty}$.

quark gluon plasma (SQGP) [13, 14, 15], the possible existence of heavy quark bound states that might survive above deconfinement [16, 17, 18, 19, 20, 21] as well as on transport properties [22] in the high temperature phase.

Here we will only discuss the asymptotic large distance behavior of these quantities, i.e. $S_{\infty}(T)=$ $S_{1}(r=\infty, T)$ and $U_{\infty}(T)=U_{1}(r=\infty, T)$. To separate entropy, $S_{\infty}$, and internal energy, $U_{\infty}$, contributions from heavy quark free energies we use standard thermodynamic relations,

$$
S_{\infty}(T)=-\frac{\partial F_{\infty}(T)}{\partial T} \quad \text { and } \quad U_{\infty}(T)=-\frac{\partial F_{\infty}(T) / T}{\partial T},
$$

to calculated their asymptotic behavior. In fig. 5 we compare internal energy (left) and entropy (right) to the asymptotic behavior of the free energy. The band at the left axis indicates the value of the energy in the zero temperature limit and the line in the right figure indicates that entropy, $T S_{\infty}$, vanishes at zero temperature.

In contrast to the monotonic decrease observed for $F_{\infty}$, both $U_{\infty}$ and $T S_{\infty}$ show qualitative different (critical) behavior in the transition region. While at small temperatures they rapidly approach values close to their zero temperature limits, both show a pronounced peak, $U_{\infty}\left(T \simeq T_{c}\right) \simeq 4.5 \mathrm{GeV}$ and $T S_{\infty}\left(T \simeq T_{c}\right) \simeq 4 \mathrm{GeV}$, which again decreases rapidly toward smaller values and become rather flat above $1.5 T_{c}$. Note that the temperature where both observables attain their maximum is in good agreement with the critical temperature obtained using different observables in [2]. Similar behavior was already observed in previous studies with $n_{f}=2$ and 3 with larger quark masses in [6, 23].

\section{Effective running coupling constant}

As already discussed, the heavy quark-antiquark free energies become temperature independent at small separations. To analyze the onset of temperature effects it is more appropriate to study the effective distance and temperature dependent running coupling defined through

$$
\alpha_{\mathrm{eff}}(r, T) \equiv \frac{3}{4} r^{2} \frac{d F_{1}(r, T)}{d r} .
$$



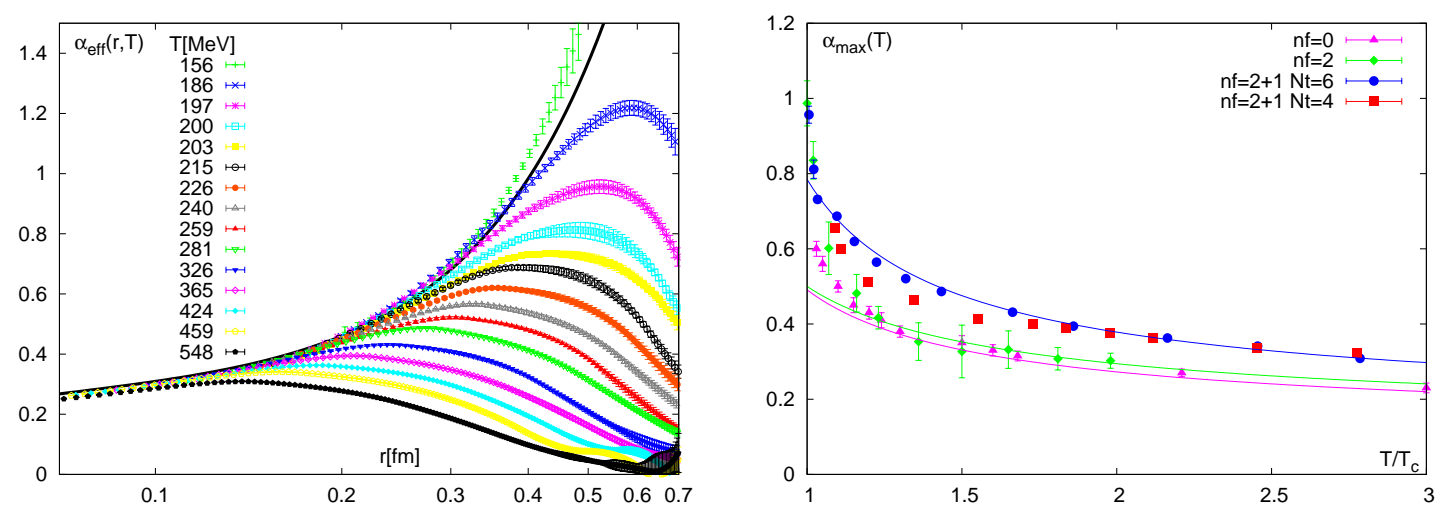

Figure 6: The effective (distance dependent) running coupling (left). The solid line shows the zero temperature running coupling calculated from the zero temperature potential. Effective temperature dependent coupling (right), $\alpha_{\max }(T)$, define by the maximum of $\alpha_{\mathrm{eff}}(r, T)$ as explained in the text for (2+1) flavor compared to 2-flavor and pure gauge theory [4, 5, 6]. The lines show a perturbative inspired fit-Ansatz for high temperatures.

In fig. 6 (left) results for various temperatures are compared to the zero temperature running coupling, $\alpha_{T=0}(r)$, defined in the same way (solid line). Note that the quadratic rise of $\alpha_{T=0}$ is a non-perturbative effect that stems from the linear rising string tension term in the potential, while at small distances the logarithmic weakening of the coupling is visible and at sufficiently small distances it should reach the perturbative (asymptotic free) behavior.

At finite temperatures, $\alpha_{\mathrm{eff}}(r, T)$ follows this zero temperature behavior to relatively large distances, before screening sets in leading to a maximum and a decrease at larger distances. Although the onset of temperature effects and the maximum is shifted towards smaller distances with increasing temperature, at temperatures slightly above the critical one, $\alpha_{\text {eff }}(r, T)$ still follows the quadratic behavior quite far, indicating that even above $T_{c}$ remnants of confinement forces are present.

We have used the value of the maximum of the running coupling, $\alpha_{\max }(T)$, to define an effective temperature dependent coupling constant that can be used to indicate an effective coupling strength at distances where the screening of the static quark-antiquark pair sets in. The results are shown in fig. 6 (right) in comparison with pure SU(3) gauge theory and 2-flavor QCD results from [5]. The solid lines show results of a fit with an Ansatz using the two-loop perturbative coupling at temperatures $T \geq 1.2 T_{c}$. Note that the 2-flavor results are calculated for rather large quark masses $(m / T=0.4)$. Therefore the increase of $\alpha_{\max }$ for $(2+1)$-flavor is rather a quark mass effect than a flavor effect. The comparison of the results for temporal extent $N_{\tau}=4$ and 6 again shows that cut-off effects also in this observable are quite small. The large value of the coupling close to the critical temperature should not be identified with a large Coulombic coupling but clearly includes non-perturbative effects.

\section{Debye screening at finite temperature and density}

We follow the commonly used approach to define the non-perturbative (Debye) screening mass, $m_{D}(T)$, and temperature dependent coupling constant, $\alpha(T)$, using a screened Coulomb fit 

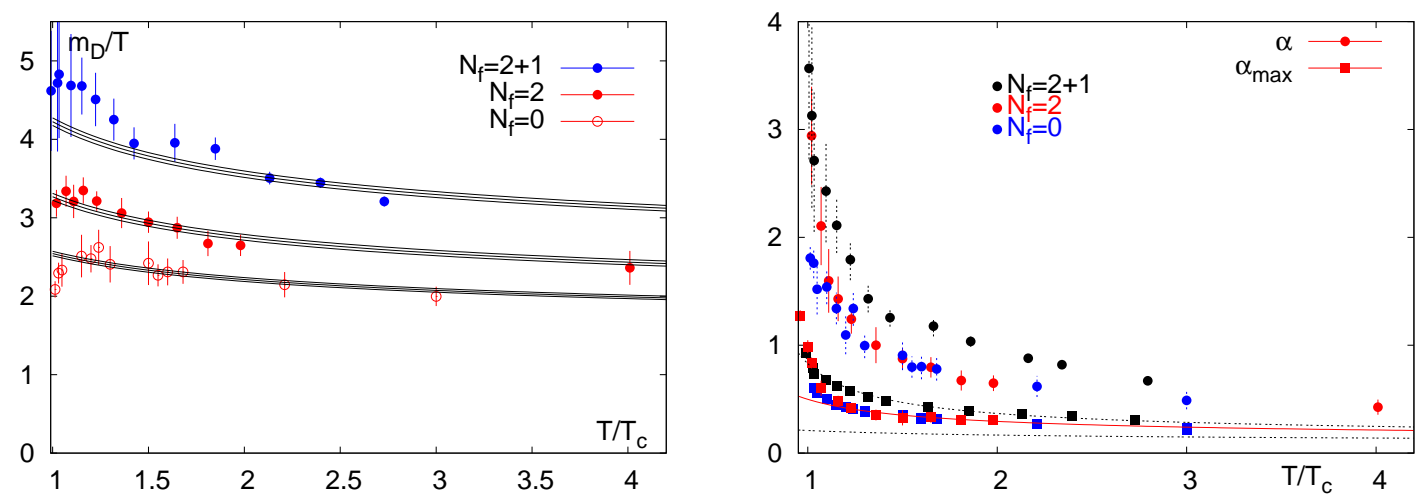

Figure 7: Debye screening masses in units of the temperature (left) for different number of flavors. The solid lines and error band show the fit of a perturbative Ansatz including a scale factor $A$ as described in the text. Effective T-dependent Coulombic couplings (right) as obtained from the same fits compared to $\alpha_{\max }$.

Ansatz for the large distance part of the heavy quark free energies normalized by its asymptotic large distance value,

$$
F_{1}(r, T)-F_{1}(r=\infty, T)=-\frac{4}{3} \frac{\alpha(T)}{r} e^{-m_{D}(T) r} .
$$

Our results for the screening masses are summarized in fig. 7 (left) as function of $T / T_{c}$ and are compared to results in pure gauge theory and 2-flavor QCD from [5].

Although we are not expecting perturbation theory to hold at the small temperatures analyzed here, the enhancement for increasing number of flavors is in qualitative agreement with leading order perturbation theory, i.e.

$$
\frac{m_{D}(T)}{T}=A\left(1+\frac{N_{f}}{6}\right)^{1 / 2} g(T) .
$$

Here we have already introduced a multiplicative constant $A$ to allow for non-perturbative contributions ( $A=1$ in perturbation theory). Using a two-loop perturbative definition of $g(T)$ a best fit analysis for $m_{D}(T) / T$ for temperatures $T \geq 1.2 T_{c}$ leads to $A=1.52(2), 1,42(2)$ and 1.66(2) for $N_{F}=0,2$ and $(2+1)$, respectively. The results including an error band are shown by the solid lines in fig. 7 (left).

The large value of $A$ indicates that non-perturbative contributions are important in the temperature range analyzed here. Note that a study in pure gauge theory up to temperatures as high as $24 T_{c}$ led to an only slightly smaller value, $A=1.39(2)$, revealing that even at such high temperatures the screening mass still is far from being perturbative.

The results for the coupling, $\alpha(T)$, are shown in fig. 团(right) in comparison with $\alpha_{\max }$ as discussed in the previous section. The large values of $\alpha(T)$, especially close to the transition region, describes the large distance behavior of heavy quark free energies and should not be confused with the coupling $\alpha_{\max }$ that characterizes the short distance part of $F_{1}(r, T)$. The latter is almost temperature independent and can to some extent be described by the zero temperature coupling.

So far we have only discussed results for vanishing baryon number densities. In leading order perturbation theory, the dependence of the Debye mass on temperature and quark chemical potential 

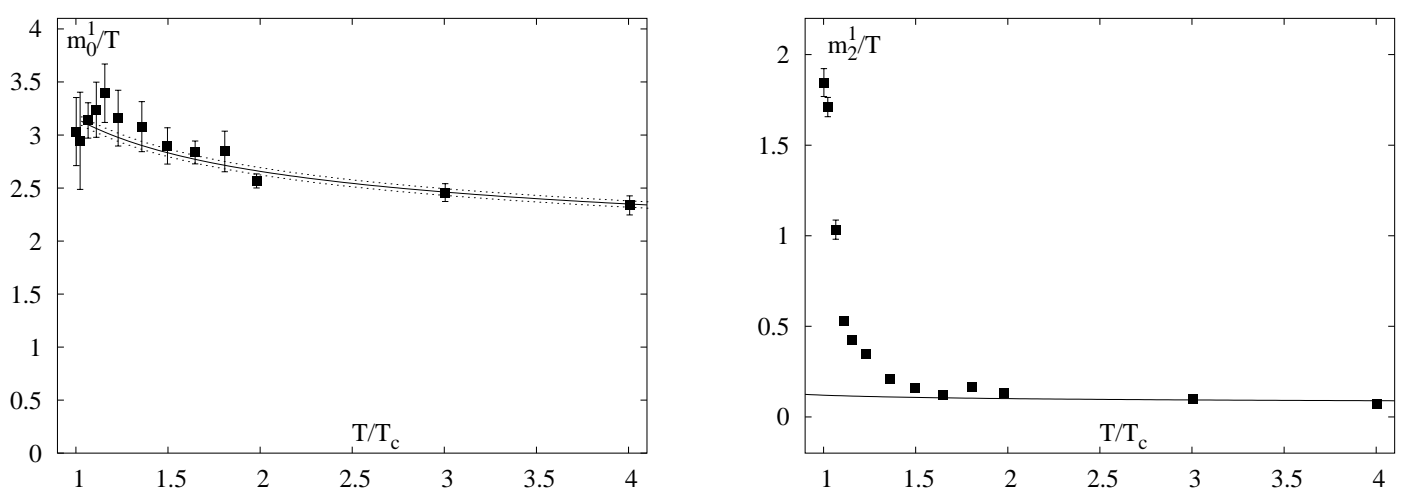

Figure 8: Zero (left) and second (right) order density coefficients of the Taylor expansion of heavy quark free energies in 2-flavor QCD [24]. The solid line in the left figure shows the fit and in the right figure the leading order perturbative result as explained in the text.

is given by

$$
\frac{m_{D}\left(T, \mu_{q}\right)}{T}=g(T) \sqrt{1+\frac{N_{f}}{6}+\frac{N_{f}}{2 \pi^{2}}\left(\frac{\mu_{q}}{T}\right)^{2}} .
$$

Here we will discuss results for two-flavor QCD with large quark masses [24]. The extension to non-zero densities in terms of a Taylor expansion in the quark chemical potential is straightforward and comparable to the Taylor expansion for bulk thermodynamic observables [25],

$$
m_{D}\left(T, \mu_{q}\right)=m_{0}(T)+m_{2}(T)\left(\frac{\mu_{q}}{T}\right)^{2}+\mathscr{O}\left(\mu_{q}^{4}\right),
$$

where $m_{0}(T)$ is the zero-order (zero density) contribution as already discussed above and $m_{2}(T)$ is the second order Taylor coefficient. Note that for a $\bar{q} q$-system the odd order coefficients vanish.

The results for $m_{0}(T)$ and $m_{2}(T)$ are shown in fig. 8 (left) and (right), respectively. The solid line in the left figure shows the fit result for $m_{0}$ as discussed above and the line in the right figure shows the second order expansion coefficient from the perturbative leading order expression (7.3). While the zero order coefficient clearly shows non-perturbative behavior up to high temperatures, the good agreement of the second order coefficient with the perturbative expectation at temperatures above $1.5 T_{c}$ indicates that non-perturbative contributions to screening in the high temperature phase of QCD are dominated by the gluonic sector.

The density dependence of screening masses so far was only analyzed for 2-flavor QCD and large quark masses. For a more realistic description the analysis will be carried out for $(2+1)$-flavor QCD with almost realistic quark masses in the future.

\section{Conclusions}

We have analyzed heavy quark free energies, their temperature dependence and screening properties for (2+1)-flavor QCD with almost realistic quark masses. The renormalization of the free energies as well as the Polyakov loop was performed using renormalization constants obtained at zero temperatures. For the asymptotic large distance behavior, entropy and internal energy contributions were separated from the free energies and show critical behavior around the transition 
region.

From the analysis of the effective running coupling constants the onset of medium effects on a heavy quark-antiquark pair was analyzed and from the large distance behavior of the free energies we extracted screening properties at vanishing density. The extension to finite baryon densities so far is limited to the case of 2-flavor QCD and large quarks masses. The agreement of the second order expansion coefficient with perturbation theory at temperatures above $1.5 T_{c}$ indicates that the main non-perturbative contributions to screening stems from the gluonic sector while fermionic contributions seem to be small. This behavior has to be confirmed for (2+1)-flavor QCD with almost realistic quark masses in the future.

\section{References}

[1] RBC-Bielefeld collaboration, in preparation.

[2] M. Cheng et al. [RBC-Bielefeld collaboration], Phys. Rev. D 74, 054507 (2006) and arXiv:0710.0354.

[3] A. Gray et al., Phys. Rev. D 72, 094507 (2005).

[4] O. Kaczmarek et al., Prog. Theor. Phys. Suppl. 153 (2004) 287.

[5] O. Kaczmarek and F. Zantow, Phys. Rev. D 71, 114510 (2005).

[6] O. Kaczmarek and F. Zantow, hep-lat/0506019.

[7] O. Philipsen, Phys. Lett. B 535, 138 (2002).

[8] O. Jahn and O. Philipsen, Phys. Rev. D 70, 074504 (2004).

[9] O. Kaczmarek, F. Karsch, P. Petreczky and F. Zantow, Phys. Lett. B 543, 41 (2002).

[10] Y. Aoki, Z. Fodor, S. D. Katz and K. K. Szabo, Phys. Lett. B 643, 46 (2006).

[11] E. Gava and R. Jengo, Phys. Lett. B 105 (1981) 285.

[12] P. Petreczky, Eur. Phys. J. C 43, 51 (2005)

[13] J. Liao and E. Shuryak, arXiv:0706.4465.

[14] E. Shuryak, Prog. Part. Nucl. Phys. 53, 273 (2004).

[15] M. Gyulassy and L. McLerran, Nucl. Phys. A 750, 30 (2005).

[16] S. Digal, P. Petreczky and H. Satz, Phys. Lett. B 514, 57 (2001), Phys. Rev. D 64, 094015 (2001)

[17] A. Mocsy and P. Petreczky, Phys. Rev. D 73, 074007 (2006) and arXiv:0705.2559, arXiv:0706.2183.

[18] D. Cabrera and R. Rapp, hep-ph/0611134.

[19] Y. Park, K. I. Kim, T. Song, S. H. Lee and C. Y. Wong, arXiv:0704.3770.

[20] W. M. Alberico, A. Beraudo, A. De Pace and A. Molinari, arXiv:0706.2846.

[21] E. V. Shuryak and I. Zahed, Phys. Rev. D 70, 054507 (2004).

[22] H. van Hees, M. Mannarelli, V. Greco and R. Rapp, arXiv:0709.2884.

[23] P. Petreczky and K. Petrov, Phys. Rev. D70 (2004) 054503.

[24] M. Doring, S. Ejiri, O. Kaczmarek, F. Karsch and E. Laermann, Eur. Phys. J. C 46, 179 (2006).

[25] C. R. Allton et al., Phys. Rev. D 71, 054508 (2005). 